\title{
Maternal hypoxia increases hippocampal cell susceptibility to ischemia after middle cerebral artery occlusion in rat offspring
}

\author{
Lingxing Wang ${ }^{1}$, Honghong Huang ${ }^{1 *}$, Hongchao Cai ${ }^{2}$, Yafang Chen ${ }^{1}$ \\ ${ }^{1}$ Department of Neurology, Second Affiliated Hospital of Fujian Medical University, Quanzhou 362000, Fujian, ${ }^{2}$ Department \\ of Neurology, Shishi (Overseas Chinese) Hospital, Shishi 362700, Fujian, China
}

\begin{abstract}
Introduction: Maternal hypoxia induces an adverse uterine environment and may induce long-term effects in offspring. This study investigated whether maternal hypoxia increases hippocampal cell vulnerability and exacerbates neurological impairments in adult rat offspring following ischemia.

Material and methods: Pregnant Sprague-Dawley rats were randomly assigned to no maternal hypoxia or maternal hypoxia treatment groups. Adult male rat offspring were subjected to middle cerebral artery occlusion (MCAO). There were four groups: maternal + sham ( $M H+$ Sham), sham (Sham), maternal hypoxia + MCAO (MH + MCAO), and MCAO only (MCAO). Neurological deficits were evaluated. Hippocampal cell damage was observed by hematoxylin and eosin (HE) staining. Cell apoptosis in the hippocampus was detected by TdT-mediated dUTP-biotin nick-end labeling (TUNEL) staining. Caspase-3, cytochrome c, Bax, and bcl-2 expression in the hippocampus was detected by Western blot.

Results: More severe hippocampal cell damage was found in the MH + MCAO group than in the MCAO group. Additionally, neurological deficits, percentage of TUNEL positive cells, and expression of caspase-3, cytochrome $c$, and Bax in the hippocampus were significantly higher $(p<0.05)$, whereas bcl-2 expression was significantly lower $(p<0.05)$ in the $M H+M C A O$ group compared to the MCAO group.

Conclusions: These findings suggest that maternal hypoxia may exacerbate hippocampal cell apoptosis in rat offspring after MCAO via alterations in the expression of cytochrome c, caspase-3, Bax, and bcl-2, which ultimately affects ischemic stroke prognosis. To our knowledge, this is the first study demonstrating that maternal hypoxia increases hippocampal cell susceptibility to ischemia in adult rat offspring.
\end{abstract}

Key words: maternal hypoxia, offspring, cerebral ischemia, hippocampus, disease susceptibility.

\section{Introduction}

Stroke is caused by the loss of circulation to a portion of the brain, which results in a sudden, focal deficit of neurological function. It is the leading cause of disability and death worldwide [14]. After a stroke, approximately $50-70 \%$ of survivors are unable to take care of themselves, whereas $15-30 \%$ of survivors remain permanently disabled. Stroke is a common disease that has destructive effects on patients and their families; thus, it is extremely important to predict the prognosis and identify the factors that affect stroke outcome. Our previous studies have demonstrated that maternal hypoxia leads to 
low birth weight [18] and pathologic changes of leukoaraiosis in adult offspring rats [17]. Since low birth weight is associated with an increased risk of stroke [12] and leukoaraiosis is associated with functional outcome after ischemic stroke [11], we might speculate that the maternal hypoxia predisposes adult rat offspring to more severe cerebral damage in response to ischemic stroke. To study that, two animal models, maternal hypoxia and focal cerebral ischemia, were used to determine the underlying mechanisms and impact of maternal hypoxia on infarct volume, neurological deficits, and hippocampal cell apoptosis in rat offspring after ischemia. To our knowledge, this is the first study to clarify the effects of maternal hypoxia on hippocampal cells after middle cerebral artery occlusion (MCAO) in adult rat offspring.

\section{Material and methods}

\section{Animals and maternal hypoxia protocol}

All experimental procedures were approved by the Animal Care and Use Committee of Fujian Medical University, China and adhered to the China National Institutes of Health guidelines (approval number: SYXK (Min) 2013-001; 17 January 2013). Virgin female Sprague-Dawley rats, which weighed 200-250 g, were purchased from the Shanghai SLAC Laboratory Animal Center, China. The animal room was maintained at a controlled temperature $(22 \pm$ $1^{\circ} \mathrm{C}$ ) with free access to water and food; the lights were turned on from 07:00 to 19:00 h. Female rats were mated with mature males, and vaginal smears were collected each morning. Pregnancy was considered when sperm-positive vaginal smears were present (day 0 ). Twenty pregnant rats were randomly assigned to maternal hypoxia treatment $(n=10)$ or no maternal hypoxia treatment $(n=10)$ groups.

Maternal hypoxia was induced as previously described [17]. In brief, pregnant rats were put into a Plexiglas chamber ( 140 I vol.) for $3 \mathrm{~h}$ per day from days 7 to 21 of pregnancy. Compressed air and nitrogen were infused into the chamber continuously to maintain the oxygen concentration at $10 \pm 1 \%$, which was monitored by a portable gas analyzer (S-450, IST-AIM). The expired $\mathrm{CO}_{2}$ and water were eliminated as previously described [17]. Maternal arterial blood gas analysis was performed to verify the hypoxic rat model by drawing blood samples from three randomly selected rats in each group. Control pregnant rats were placed in an identical chamber, in which only compressed air was infused, for the same time during gestation. After birth, the litters of the rats were adjusted to eight pups per dam to standardize postnatal nutrition. The pups were weaned at 21 days, and only male offspring (three to four males per litter) were evaluated in subsequent experiments.

\section{Rat MCAO model}

Adult male offspring rats (250-270 g) underwent transient focal cerebral ischemia using MCAO as previously described [9]. Briefly, the rats were anesthetized with $10 \%$ chloral hydrate $(400 \mathrm{mg} / \mathrm{kg})$ via intraperitoneal injection. The right common carotid, right external carotid, and internal carotid arteries were subsequently separated and exposed. A 4-0 monofilament with poly-L-lysine coated blunt end was gently inserted into the right internal carotid artery through the external carotid artery to block the origin of the right MCA. After $60 \mathrm{~min}$, the filament was slowly removed to enable reperfusion. The body temperature was maintained at $37.0-37.5^{\circ} \mathrm{C}$ throughout the surgery with a heating blanket. Adult male rats in the sham group were subjected to the same operation procedures with the exception of MCAO. The rats were randomly divided into four groups: maternal hypoxia + sham (MH + Sham); sham (Sham); maternal hypoxia + MCAO (MH + MCAO); and MCAO only (MCAO) $(n=10$ per group).

\section{Neurological assessment}

After $24 \mathrm{~h}$ of reperfusion, neurological examinations were performed by an observer blind to the study using a five-point scale [9] as follows: 0 - no apparent neurological defect; 1 - unable to extend left forelimb; 2 - circling to the left; 3 - falling to the left; and 4 - failure to spontaneously walk and a depressed level of consciousness.

\section{Tissue collection and infarct volume measurement}

After neurological assessment, five rats per group were transcardially perfused with saline, followed by $4 \%$ neutral-buffered paraformaldehyde under deep anesthesia. The brains were placed in $10 \%$ formalin overnight following removal from the skulls and processed in graded alcohols and xylene. Finally, the brains were embedded in paraffin for sectioning. 
Infarct size was measured by hematoxylin and eosin (HE) staining as previously described [3]. Briefly, five coronal brain sections, each approximately $2 \mathrm{~mm}$ apart, were stained with HE. Images were captured with a camera and quantified with NIH Image J software (http://rsb.info.nih.gov/nih-image/download html) by a blinded observer. Infarct volumes were calculated and presented as a percentage of the ipsilateral hemisphere to correct for brain swelling. Brain cell damage was examined using light microscopy under $400 \times$ magnification.

\section{TdT-mediated dUTP-biotin nick-end labeling (TUNEL) labeling}

An in situ cell death detection kit (Roche, Mannheim, Germany) was used for TUNEL staining according to the manufacturer's instructions. Cells that contained dark brown nuclei were considered TUNEL-positive cells. TUNEL-positive cells and all cells were counted in the hippocampal cornu ammonis 1 (CA1) regions of each group using light microscopy under $400 \times$ magnification. TUNEL-positive cells were presented as a percentage of the total cells and used for statistical analyses.

\section{Western blotting}

After neurological assessment, five rats per group were deeply anesthetized and the brains were rapidly removed. The hippocampus ipsilateral to the occluded side was separated and flash-frozen in liquid nitrogen. The hippocampal tissues were homogenized in RIPA buffer (Biosynthesis Biotechnology Company, Beijing, China). The crude homogenates were centrifuged for $15 \mathrm{~min}$ at $10,000 \times \mathrm{g}$, and the supernatant protein concentration was determined using the BCA method (Pierce Chemical Co., Rockford, IL, USA). The supernatant was mixed with loading buffer and boiled for 3 min. Protein $(50 \mu \mathrm{g})$ was separated by electrophoresis and transferred onto nitrocellulose membranes. After a $1 \mathrm{~h}$ block in 5\% bovine serum albumin at room temperature, the membrane was incubated with primary antibodies against caspase-3 (Cell Signaling Technology, Beverly, MA, USA, 1 : 500), cytochrome c (Santa Cruz Biotechnology Inc., CA, USA, 1 : 1000), Bax (Cell Signaling Technology, Beverly, MA, USA, 1 : 500), Bcl-2 (Santa Cruz Biotechnology Inc., CA, USA, 1 : 200), and $\beta$-actin (Biosynthesis Biotechnology Company, Beijing, China, $1:$ 2000). The membranes were subsequently incubated with peroxidase-conjugated secondary antibody (Santa Cruz Biotechnology Inc., CA, USA, $1: 5000)$, and protein bands were visualized using an ECL kit (Biosynthesis Biotechnology Company, Beijing, China). The bands were digitized and quantified using Quantity One software (Bio-Rad, Hercules, CA, USA). The protein signals were normalized to $\beta$-actin.

\section{Statistical analysis}

All statistical analyses were conducted using SPSS 16.0 software (SPSS Inc., Chicago, IL, USA). Neurological deficit scores are expressed as the median values (quartile range $25-75 \%$ ), and the Mann-Whitney $U$ test was used for the analysis. Other data are presented as the mean \pm standard error of the mean (SEM). Unpaired Student's $t$ test was used when only two groups were involved. One-way analysis of variance (ANOVA) was used for comparisons of data with more than two groups, followed by the Student-Newman-Keuls (SNK) post hoc test. $P<0.05$ was considered statistically significant.

\section{Results}

\section{Maternal arterial blood gas analysis}

Maternal blood $\mathrm{PaO}_{2}$ and $\mathrm{SaO}_{2}$ were significantly lower in the maternal hypoxia treatment group than in the no hypoxia treatment group ( $p<0.05$; Table I). There was no significant difference in blood $\mathrm{PH}$ or $\mathrm{PaCO}_{2}$ between the maternal hypoxia treatment group and no hypoxia treatment group (Table I).

\section{Behavioral scores}

No neurological impairment was observed in the Sham or $\mathrm{MH}+$ Sham group. After $24 \mathrm{~h}$ of reperfusion,

Table I. Results of maternal arterial blood gas analysis

\begin{tabular}{|lllll|}
\hline Group $(n=3)$ & $\mathrm{PaO}_{2}(\mathrm{kPa})$ & $\mathrm{SaO}_{2}(\%)$ & $\mathrm{pH}$ & $\mathrm{PaCO}_{2}(\mathrm{kPa})$ \\
\hline Maternal hypoxia treatment group & $7.37 \pm 0.69^{*}$ & $74.11 \pm 0.12^{*}$ & $7.37 \pm 0.01$ & $6.36 \pm 1.65$ \\
\hline No hypoxia treatment group & $11.20 \pm 0.57$ & $97.01 \pm 1.23$ & $7.42 \pm 0.03$ & $5.71 \pm 0.97$ \\
\hline
\end{tabular}

Values are expressed as mean \pm SEM.

${ }^{*} p<0.05$ compared with no hypoxia treatment group. 
neurological symptoms were apparent in the MCAO and $\mathrm{MH}+\mathrm{MCAO}$ groups. Furthermore, maternal hypoxia significantly increased the neurological deficits in the $\mathrm{MH}+\mathrm{MCAO}$ group. The median (25-75\% quartile range) neurological deficit in the MCAO group was 2 (1.75-2.25) compared with 3 (2.00-3.00) in the $\mathrm{MH}+\mathrm{MCAO}$ group $(p<0.05)$.

\section{Infarct size}

Representative HE stained coronal brain sections are shown in Figure 1. Infarction was identified in the MCAO and $\mathrm{MH}+\mathrm{MCAO}$ groups, but not in the Sham and $\mathrm{MH}+$ Sham groups. The rats in the MCAO group and $\mathrm{MH}+\mathrm{MCAO}$ group both exhibited cortical and subcortical infarction, as well as striatal and hippocampal infarctions (Fig. 1A). The infarct volume was significantly higher in the $\mathrm{MH}+$ MCAO group (30.4 $\pm 3.2 \%, n=5)$ than the MCAO group $(19.0 \pm 2.0 \%$, $n=5 ; p<0.05)$ (Fig. 1B).

\section{Brain cell damage and apoptosis}

In the MCAO rats, brain cell damage was histologically identified in the hippocampal CA1 region; however, there was no brain cell damage in the

A
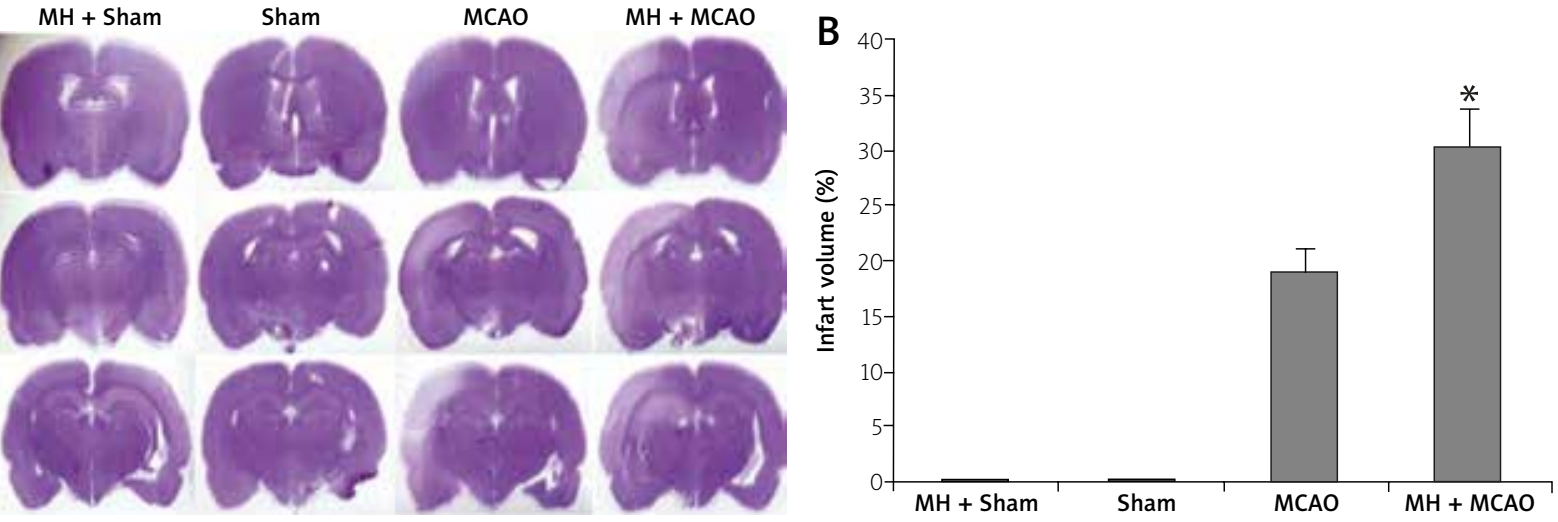

C
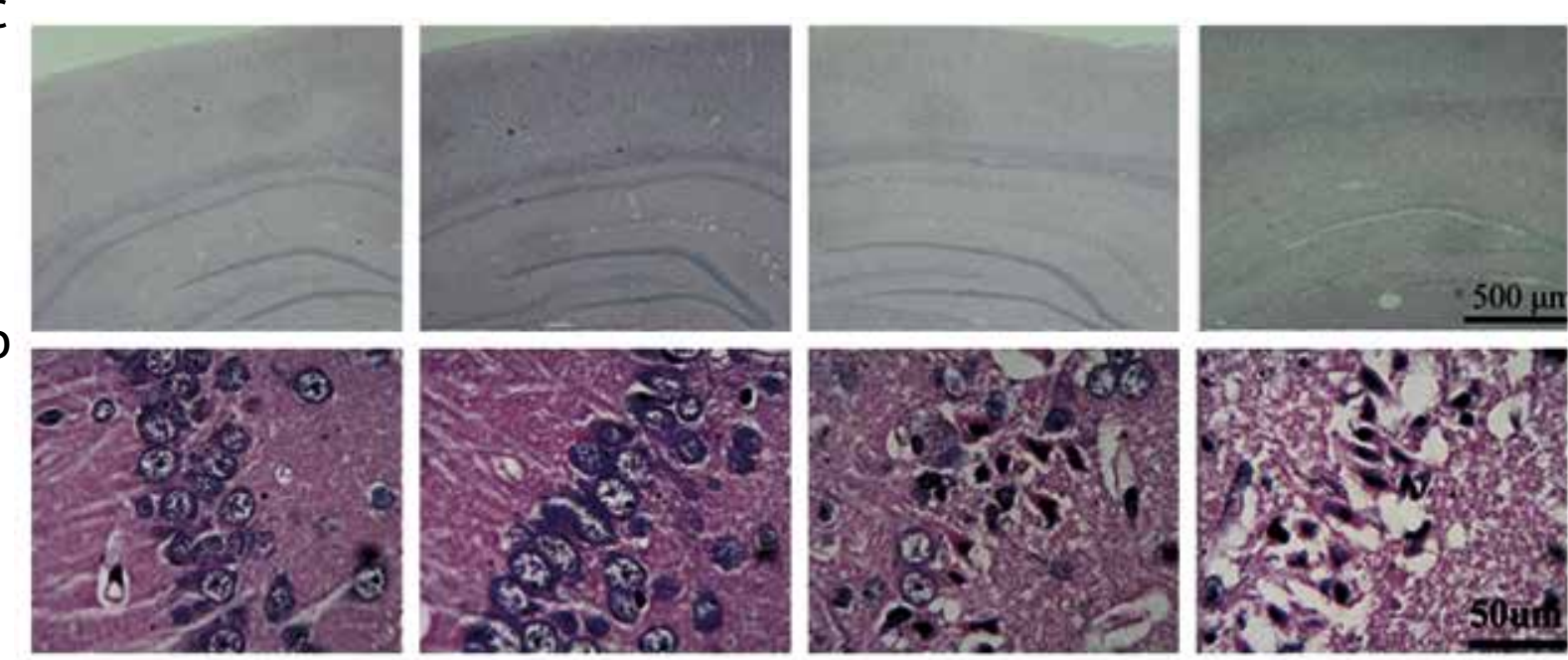

Fig. 1. Representative photographs of HE stained brain sections obtained from experimental groups after transient middle cerebral artery occlusion (MCAO). A) HE staining of infarction in $\mathrm{MH}+$ Sham, MCAO, and $\mathrm{MH}$ + MCAO groups. B) Quantified data showing a significant increase in the infarct area in the $M H+M C A O$ group compared to the MCAO group. No infarct area was identified in the $\mathrm{MH}+$ Sham group. Data are expressed as mean \pm SEM * $p<0.05$ vs. the MCAO group. C) MCAO resulted in brain cell damage in the hippocampal CA1 region ipsilateral to ischemia, and maternal hypoxia exacerbated brain cell damage in the hippocampal CA1 region and the dentate gyrus. 
Sham and $M H+$ Sham groups. Furthermore, the $\mathrm{MH}+\mathrm{MCAO}$ group exhibited more extensive brain cell damage than the MCAO group (Fig. $1 C$ and D). Few TUNEL-positive apoptotic cells were observed in the hippocampal CA1 region in the Sham and $\mathrm{MH}+$ Sham groups (Fig. 2A-D). The MCAO group $(42.0 \pm 3.8 \%, n=5)$ had a significantly higher percentage of TUNEL-positive cells in the hippocampal CA1 region compared with the Sham group (2.5 \pm $0.9 \%, n=5 ; p<0.05)$. The percentage of TUNELpositive cells was significantly higher in the $\mathrm{MH}+$ MCAO group $(60.0 \pm 5.6 \%, n=5)$ than in the MCAO group in the hippocampal CA1 region $(p<0.05$; Fig. 2E).

\section{Caspase-3, cytochrome c, Bax, and $\mathrm{BCl}-2$ expression}

Expression of cytoplasmic caspase-3, cytochrome c, and Bax in the hippocampal CA1 region was significantly up-regulated in the MCAO group compared with the Sham group $(p<0.05)$. Moreover, the levels of cytoplasmic cytochrome c, caspase-3, and Bax were significantly higher in the $\mathrm{MH}+\mathrm{MCAO}$ group compared with the MCAO group ( $p<0.05$; Fig. $3 A$ ). $\mathrm{BCl}-2$ expression in the hippocampal CA1 region of the MCAO group was significantly downregulated compared to the Sham group $(p<0.05)$. Furthermore, Bcl-2 expression was lower in the $\mathrm{MH}+\mathrm{MCAO}$ group compared with the MCAO group ( $p<0.05$; Fig. 3B).
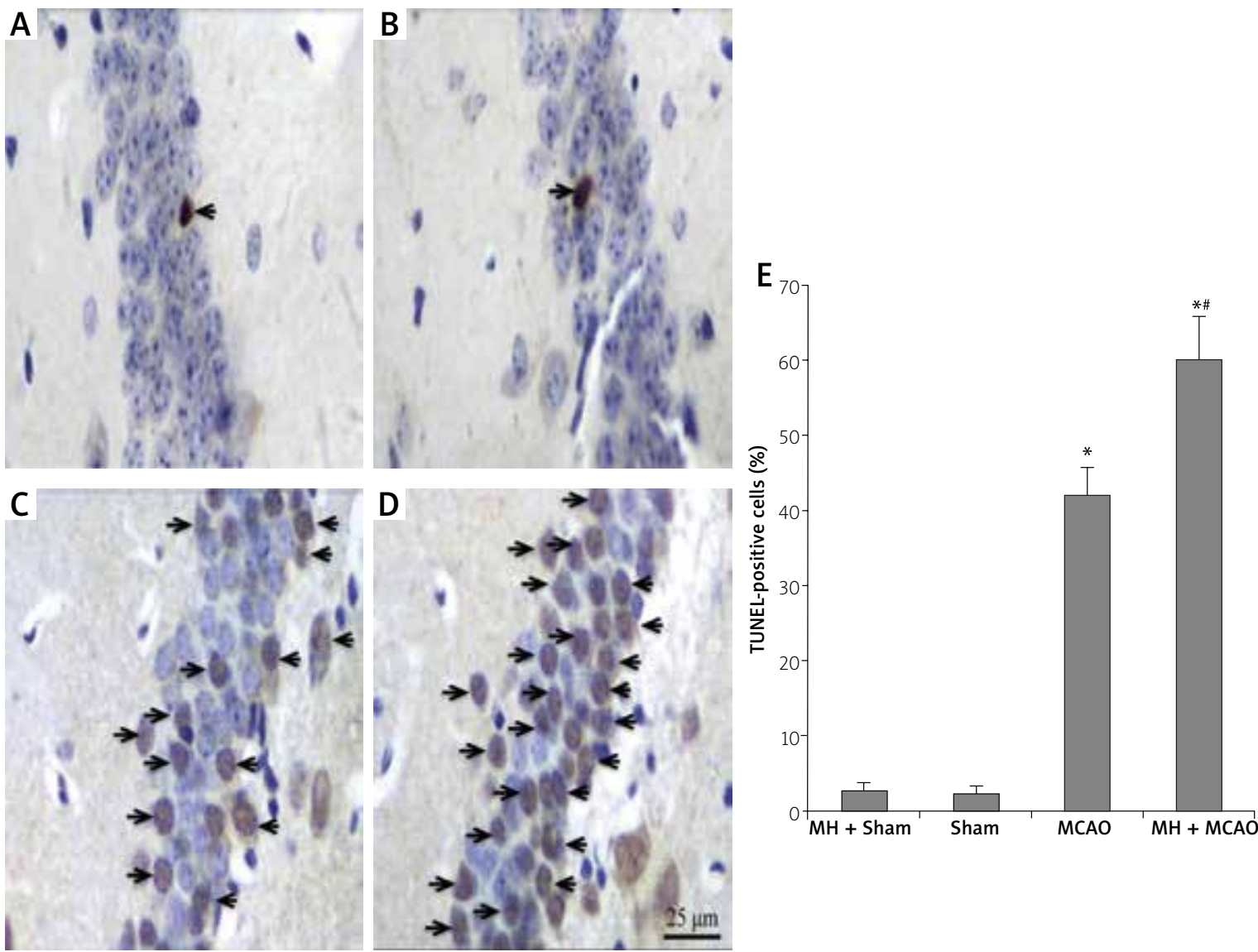

Fig. 2. TUNEL staining in the ipsilateral hippocampal CA1 region after transient middle cerebral artery occlusion (MCAO). A-D) Representative photomicrographs of TUNEL-positive cells obtained from four experimental groups. A) $\mathrm{MH}+$ Sham group; B) Sham group; C) MCAO group; and D) MH + MCAO group. E) Quantitative analysis identified an increased percentage of TUNEL-positive cells in the MCAO group compared to the Sham group. The MH + MCAO group exhibited a significantly increased percentage of apoptotic cells compared to the MCAO group. ${ }^{*} p<0.05$ vs. the Sham group; ${ }^{\#} p<0.05$ vs. the MCAO group. 


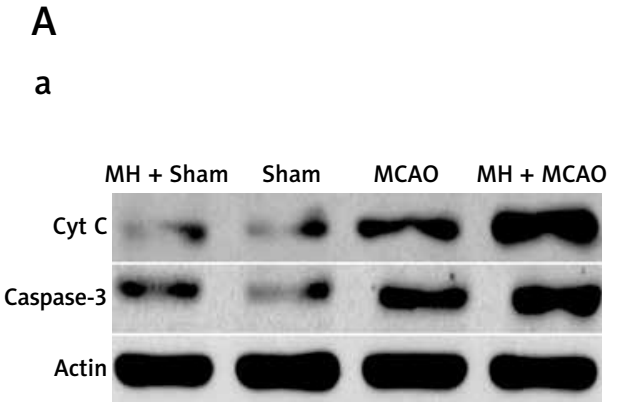

B

a

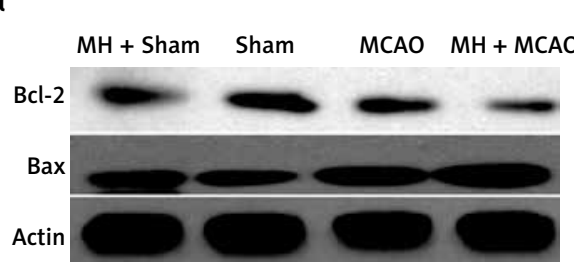

b

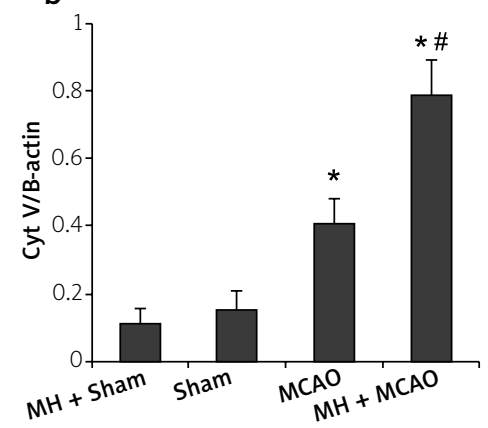

b

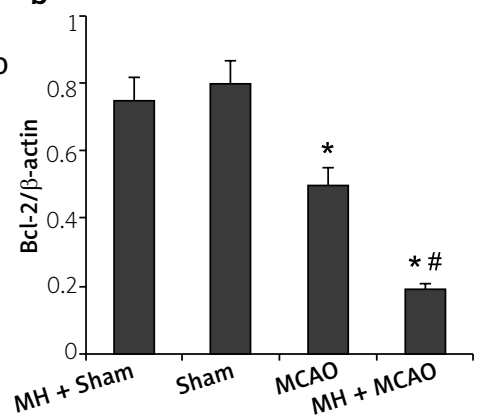

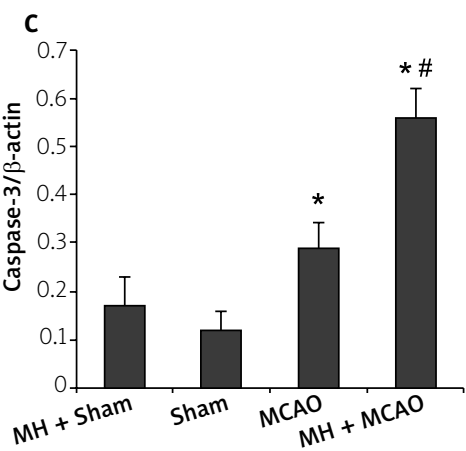

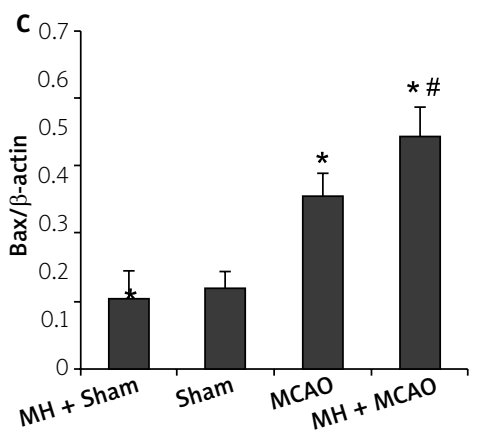

Fig. 3. Cytochrome c, caspase-3, Bax, and Bcl-2 expression after transient MCAO. A) Densitometry analysis shows that cytochrome $c$ and caspase- 3 expression was upregulated in the MCAO group compared to the Sham group. Maternal hypoxia significantly increased cytochrome $c$ and caspase- 3 expression in the $\mathrm{MH}+\mathrm{MCAO}$ group compared to the MCAO group. B) MCAO significantly upregulated Bax expression and downregulated $\mathrm{BCl}-2$ expression in the ischemic rats. Bax expression was significantly increased in the $\mathrm{MH}$ + MCAO group compared to the MCAO group, and Bcl-2 expression was significantly decreased in the $\mathrm{MH}$ + MCAO group compared to the MCAO group. ${ }^{*} p<0.05$ vs. the Sham group; ${ }^{*} p<0.05$ vs. the MCAO group.

\section{Discussion}

The results of maternal arterial blood gas analysis indicated that there was significant hypoxemia without retention of $\mathrm{CO}_{2}$ and acidosis, and it was a suitable model for the study of maternal hypoxia. Moreover, our data demonstrated that the $\mathrm{MH}$ + MCAO group exhibited more severe neurological deficits and a larger infarct size compared to the MCAO group. These findings indicate that the effect of maternal hypoxia persists postnatally and increases vulnerability to cerebral ischemia-reperfusion.

Blood supply to the hippocampus originates from several arteries, in particular the posterior cerebral and anterior choroidal arteries. Therefore, the hippocampus is considered a penumbra type of injury in the rat model of MCAO [7]. Apoptosis is one of the major cell death patterns in this ischemic penumbra [4]. Consistent with a previous finding [20], our results demonstrated that MCAO contributed to cell apoptosis in the ipsilateral hippocampal region following ischemia. Furthermore, our data also showed that maternal hypoxia did not directly lead to obvious hippocampal cell apoptosis, but it exacerbated brain cell damage and intensified apoptosis in the hippocampus of rat offspring exposed to cerebral ischemia. Since apoptosis plays a crucial role in cell death after cerebral ischemia and focal ischemia leads to remarkable tissue loss because of apoptotic cells, we speculate that increased apoptosis explains why maternal hypoxia results in deteriorated neurological outcomes after MCAO in rat offspring.

Following cerebral ischemia, cytochrome $c$ is released from mitochondria into the cytosol, which 
subsequently triggers caspase- 3 activation and causes cytoskeleton degradation, ultimately resulting in DNA fragments and cell death [2]. The cytochrome c/ caspase-3 apoptotic pathway, which is considered an energy-dependent active process, occurs only in the ischemic penumbra because of the residual blood supply [1]. Thus, we measured caspase- 3 and cytochrome $c$ expression in the hippocampus ipsilateral to ischemia. Previous studies have demonstrated that caspase-3 expression increases after stroke [20]. Deletion or inhibition of caspase- 3 induces neuroprotective effects via a reduction in cerebral infarct size and prolongs the therapeutic window $[6,13,20]$. In the present study, we demonstrated that MCAO increased the levels of caspase- 3 and cytochrome $\mathrm{c}$ in the rat hippocampus. Moreover, maternal hypoxia significantly upregulated cytochrome $c$ and caspase- 3 expression in rat offspring following ischemia. Thus, it is possible that maternal hypoxia exacerbates apoptosis via promoting cytochrome $c$ release and caspase- 3 expression, and the increased apoptosis contributes to the development of ischemic infarct with DNA fragmentation.

After stoke, affected cells may antagonize apoptosis via a pro-survival pathway. $\mathrm{Bcl}-2$ is regarded as an important anti-apoptotic protein [16]. Bcl-2 resides on the outer mitochondrial membrane oriented toward the cytosol, and it prevents the release of cytochrome c from the mitochondria by reducing permeabilization of the mitochondrial outer membrane [15]. Thus, Bcl-2 increases cell survival by inhibiting apoptosis. Studies have shown that decreased $\mathrm{Bcl}-2$ expression is related to apoptosis in the cerebral ischemic region [10], and over-expression of $\mathrm{Bcl}-2$ in rat cortical neurons inhibits caspase- 3 activation and defends against apoptosis-mediated cell death caused by ischemia [2]. In contrast, Bax promotes the release of cytochrome c. Thus, Bax is considered an apoptosis-promoting factor [18]. As two upstream apoptosis factors, the balance between anti-apoptotic Bcl-2 and pro-apoptotic Bax is crucial in the regulation of apoptotic cell death. In our study, ischemia significantly decreased $\mathrm{Bcl}-2$ expression and increased Bax expression in the MCAO rat group, which was consistent with a previous study [8]. Moreover, maternal hypoxia downregulated $\mathrm{Bcl}-2$ and upregulated Bax expression in the rat offspring exposed to MCAO. These findings indicate that maternal hypoxia promotes apoptosis by changing the $\mathrm{Bcl}-2 / \mathrm{Bax}$ balance.

There are several limitations in the present study. First, we only assessed one time point after MCAO.
Identifying changes in neurological function and apoptosis at different time points prior to or after MCAO may be helpful to clarify the effects of maternal hypoxia on neurodevelopment and cerebral ischemia over time, and may also facilitate determining a therapeutic window. Second, detailed neurobehavioral scoring, including memory function, should be evaluated in future studies.

\section{Conclusions}

We found that maternal hypoxia is a risk factor for stroke prognosis. Specifically, we found that maternal hypoxia increased hippocampal cell susceptibility to ischemia in adult rat offspring exposed to MCAO, with a resultant increase in apoptosis. This increased apoptosis may ultimately result in increased infarct volume and severe neurological impairment after stroke. The underlying mechanism for enhanced apoptosis may be associated with alterations in expression of cytochrome c, caspase-3, Bax and $\mathrm{Bcl}-2$, pointing towards important interventional targets to improve the functional outcome after ischemia in maternal hypoxic offspring.

\section{Acknowledgments}

This work was supported by a grant from the Natural Science Foundation of Fujian (2015J01449), Young and Middle-aged Backbone Project of Fujian Health and Family Planning Commission (2016-ZQN-53).

\section{Disclosure}

Authors report no conflict of interest.

\section{References}

1. Benchoua A, Guégan C, Couriaud C, Hosseini H, Sampaïo N, Morin D, Onténiente B. Specific caspase pathways are activated in the two stages of cerebral infarction. J Neurosci 2001; 21: 7127-7134.

2. Broughton BR, Reutens DC, Sobey CG. Apoptotic mechanisms after cerebral ischemia. Stroke 2009; 40: e331-339.

3. Czech B, Pfeilschifter W, Mazaheri-Omrani N, Strobel MA, Kahles T, Neumann-Haefelin T, Rami A, Huwiler A, Pfeilschifter J. The immunomodulatory sphingosine 1-phosphate analog FTY720 reduces lesion size and improves neurological outcome in a mouse model of cerebral ischemia. Biochem Biophys Res Commun 2009; 389: 251-256.

4. Graham SH, Chen J. Programmed cell death in cerebral ischemia. J Cereb Blood Flow Metab 2001; 21: 99-109.

5. Hwang L, Choi IY, Kim SE, Ko IG, Shin MS, Kim CJ, Kim SH, Jin JJ, Chung JY, Yi JW. Dexmedetomidine ameliorates intracerebral 
hemorrhage-induced memory impairment by inhibiting apoptosis and enhancing brain-derived neurotrophic factor expression in the rat hippocampus. Int I Mol Med 2013; 31: 1047-1056.

6. Le DA, Wu Y, Huang Z, Matsushita K, Plesnila N, Augustinack JC, Hyman BT, Yuan J, Kuida K, Flavell RA, Moskowitz MA. Caspase activation and neuroprotection in caspase-3- deficient mice after in vivo cerebral ischemia and in vitro oxygen glucose deprivation. Proc Natl Acad Sci U S A 2002; 99: 15188-15193.

7. Li JS, Zhang W, Kang ZM, Ding SJ, Liu WW, Zhang JH, Guan YT, Sun XJ. Hyperbaric oxygen preconditioning reduces ischemiareperfusion injury by inhibition of apoptosis via mitochondrial pathway in rat brain. Neuroscience 2009; 159: 1309-1315.

8. Li Z, Pang L, Fang F, Zhang G, Zhang J, Xie M, Wang L. Resveratrol attenuates brain damage in a rat model of focal cerebral ischemia via up-regulation of hippocampal Bcl-2. Brain Res 2012; 1450: 116-124.

9. Longa EZ, Weinstein PR, Carlson S, Cummins R. Reversible middle cerebral artery occlusion without craniectomy in rats. Stroke 1989; 20: 84-91

10. Miao J, Wang L, Zhang X, Zhu C, Cui L, Ji H, Liu Y, Wang X. Protective Effect of Aliskiren in Experimental Ischemic Stroke: Up-Regulated p-PI3K, p-AKT, Bcl-2 Expression, Attenuated Bax Expression. Neurochem Res 2016; 41: 2300-2310.

11. Onteddu SR, Goddeau RP, Minaeian A, Henninger N. Clinical impact of leukoaraiosis burden and chronological age on neurological deficit recovery and 90-day outcome after minor ischemic stroke. J Neurol Sci 2015; 359: 418-423.

12. Osmond C, Kajantie E, Forsén TJ, Eriksson JG, Barker DJ. Infant growth and stroke in adult life: the Helsinki birth cohort study. Stroke 2007; 38: 264-270.

13. Rami A, Sims J, Botez G, Winckler J. Spatial resolution of phospholipid scramblase 1 (PLSCR1), caspase-3 activation and DNA-fragmentation in the human hippocampus after cerebral ischemia. Neurochem Int 2003; 43: 79-87.

14. Snarska KK, Bachórzewska-Gajewska H, Kapica-Topczewska K, Drozdowski W, Chorąży M, Kułakowska A, Małyszko J. Hyperglycemia and diabetes have different impacts on outcome of ischemic and hemorrhagic stroke. Arch Med Sci 2017; 13: 100-108.

15. Tan C, Dlugosz PJ, Peng J, Zhang Z, Lapolla SM, Plafker SM, Andrews DW, Lin J. Auto-activation of the apoptosis protein Bax increases mitochondrial membrane permeability and is inhibited by Bcl-2. J Biol Chem 2006; 281: 14764-14775.

16. Tao T, Zhao M, Yang W, Bo Y, Li W. Neuroprotective effects of therapeutic hypercapnia on spatial memory and sensorimotor impairment via anti-apoptotic mechanisms after focal cerebral ischemia/reperfusion. Neurosci Lett 2014; 573: 1-6.

17. Wang L, Cai R, Lv G, Huang Z, Wang Z. Hypoxia during pregnan$c y$ in rats leads to the changes of the cerebral white matter in adult offspring. Biochem Biophys Res Commun 2010; 396: 445-450.

18. Wang Z, Huang Z, Lu G, Lin L, Ferrari M. Hypoxia during pregnancy in rats leads to early morphological changes of atherosclerosis in adult offspring. Am J Physiol Heart Circ Physiol 2009; 296: H1321-1328.

19. Zhang H, Li Q, Li Z, Mei Y, Guo Y. The protection of Bcl-2 overexpression on rat cortical neuronal injury caused by analogous ischemia/reperfusion in vitro. Neurosci Res 2008; 62: 140-146.
20. Zhang P, Zhang Y, Zhang J, Wu Y, Jia J, Wu J, Hu Y. Early Exercise Protects against Cerebral Ischemic Injury through Inhibiting Neuron Apoptosis in Cortex in Rats. Int J Mol Sci 2013; 14: 6074 6089. 